\title{
Safety and Incidence of Cardiovascular Events in Chinese Patients with Acute Coronary Syndrome Treated with Ticagrelor: the 12-Month, Phase IV, Multicenter, Single-Arm DAYU Study
}

\author{
Runlin Gao ${ }^{1}$ - Yongjian $\mathrm{Wu}^{1} \cdot$ Hengliang $\mathrm{Liu}^{2} \cdot \mathrm{Guohai}^{\mathrm{Su}}{ }^{3} \cdot$ Zuyi Yuan $^{4} \cdot$ Aidong Zhang ${ }^{5} \cdot$ Yong Wang $^{6}$. \\ Zhirong Wang ${ }^{7}$ - Yan Wang ${ }^{8}$ • Huanyi Zhang ${ }^{9} \cdot$ Yang Zheng ${ }^{10} \cdot$ Lei Liu $^{11}$ • Lijun Shen ${ }^{11}$. \\ Maria Leonsson-Zachrisson ${ }^{12}$. Yaling $\mathrm{Han}^{13}$. on behalf of the DAYU study investigators
}

Published online: 28 February 2018

(C) The Author(s) 2018. This article is an open access publication

\begin{abstract}
Purpose Ticagrelor is an orally administered, reversibly binding, direct-acting $\mathrm{P} 2 \mathrm{Y}_{12}$ receptor antagonist previously evaluated in several phase III trials. This phase IV, multicenter, single-arm trial assessed the safety and incidence of cardiovascular $(\mathrm{CV})$ events with ticagrelor in Chinese patients experiencing an acute coronary syndrome (ACS).

Methods Patients hospitalized with an ACS received ticagrelor (180 mg loading dose, $90 \mathrm{mg}$ twice daily thereafter) plus lowdose aspirin (75-100 mg/day) for up to 12 months. Safety was evaluated via PLATO-defined bleeding events, adverse events (AEs), serious AEs, and laboratory measurements. The incidence of major CV events was also evaluated.

Results The safety population included 2001 patients. During ticagrelor treatment, 426 (21.3\%) patients had at least one PLATOdefined bleeding AE, mainly minimal bleedings $(n=333)$. Major bleeding events occurred in $27(1.3 \%)$ patients, including fatal/ life-threatening bleeding in $17(0.8 \%)$ patients and other major bleeding in $11(0.5 \%)$ patients, with a Kaplan-Meier estimate of patients with the event $(95 \% \mathrm{CI})$ of $1.6 \%(1.1-2.3 \%)$. In total, $784(39.2 \%)$ patients had at least one non-bleeding AE, the majority of which were mild in severity. The composite endpoint of $\mathrm{CV}$ death, myocardial infarction, and stroke occurred in 83 (4.1\%) patients.

Conclusions Ticagrelor plus low-dose aspirin for up to 1 year was associated with a low rate of major bleeding events and a low incidence of major CV events (CV death, myocardial infarction, stroke) in Chinese patients with ACS. The overall safety profile of ticagrelor in this population was in line with current prescribing information.
\end{abstract}

Keywords Acute coronary syndrome $\cdot$ Chinese patients $\cdot$ Ticagrelor $\cdot$ Safety

Electronic supplementary material The online version of this article (https://doi.org/10.1007/s10557-018-6772-3) contains supplementary material, which is available to authorized users.

Runlin Gao

gaorunlin@citmd.com

1 Fuwai Hospital, National Center for Cardiovascular Diseases, Chinese Academy of Medical Sciences, 167 Beilishi Road, Xi Cheng District, Beijing, China

2 Zhengzhou People Hospital, Zhengzhou, China

3 Jinan Central Hospital, Jinan, China

4 First Affiliated Hospital of Medical College of Xi'An Jiaotong University, Xi'an, China

5 The First Affiliated Hospital of Jinan University, Guangzhou, China
China-Japan Friendship Hospital, Beijing, China

7 The Affiliated Hospital of Xuzhou Medical University, Xuzhou, China

8 Xiamen Heart Center, Xiamen, China

9 The Central Hospital of Tai'an, Tai'an, China

10 The First Hospital of Jilin University, Changchun, China

11 AstraZeneca China, Shanghai, China

12 AstraZeneca R\&D, Mölndal, Sweden

13 The General Hospital of Shenyang Military Region, Shenyang, China 


\section{Introduction}

Currently, 230 million individuals have cardiovascular disease (CVD) in China [1]. Chinese patients experiencing an acute coronary syndrome (ACS) — encompassing unstable angina, non-ST elevation myocardial infarction (NSTEMI), and ST elevation myocardial infarction (STEMI) - have a high mortality rate $[2,3]$. Thus, improved use of guidelinerecommended therapies in this population is required [4]. Dual antiplatelet therapy (DAPT) with aspirin and a P2 $\mathrm{Y}_{12}$ receptor inhibitor (ticagrelor, clopidogrel or prasugrel for $\geq 12$ months) represents the cornerstone of ACS management [5-9].

Ticagrelor, an orally administered, direct-acting, reversibly binding $\mathrm{P}_{2} \mathrm{Y}_{12}$ receptor antagonist, inhibits adenosine diphosphate-induced platelet aggregation [10,11] and cellular uptake of adenosine via inhibition of the equilibrative nucleoside transporter 1 [12]. Ticagrelor with low-dose aspirin is approved in more than 100 countries (including China) to reduce the rate of $\mathrm{CV}$ death, myocardial infarction (MI), and stroke in ACS patients (180 mg loading dose then $90 \mathrm{mg}$ twice daily [b.i.d] for the first year post-ACS event) [13]. In some countries and regions (e.g., USA, Japan, Hong Kong, and EU countries), ticagrelor is approved to reduce the rate of $\mathrm{CV}$ death, MI, and stroke in patients with a history of MI (60 mg b.i.d from 1 year post-MI).

The efficacy and safety of ticagrelor have been established in two, key phase III trials, PLATO in patients with ACS [14] and PEGASUS-TIMI 54 in patients with a prior MI [15]. In PLATO, ticagrelor plus aspirin significantly reduced the rate of the primary composite endpoint (MI, stroke, and death from vascular causes) in ACS patients versus clopidogrel plus aspirin [14]. Moreover, the hazard ratio (HR) (95\% confidence interval $[\mathrm{CI}])$ for cardiovascular death in PLATO was 0.79 $(0.69-0.91 ; p=0.001)$ [14]. PLATO reported no difference in the overall rate of major bleeding between ticagrelor and clopidogrel. However, compared with clopidogrel, ticagrelor was associated with a higher rate of PLATO-defined major bleeding not related to coronary artery bypass grafting (CABG), including a higher incidence of fatal intracranial bleeding but fewer instances of non-intracranial types of fatal bleeding; overall fatal bleeding was not different between ticagrelor and clopidogrel [14].

PLATO and PEGASUS-TIMI 54 were conducted worldwide, and recruited some Asian patients [14, 15]. In PLATO, 587 ACS patients of Chinese ethnicity were enrolled (i.e., $3.1 \%$ of 18,758 enrolled patients) and 577 were randomized and received study treatment. Of these Chinese patients, 418 were enrolled and 416 were randomized in Mainland China [AstraZeneca data on file]. In a retrospective PLATO analysis, the efficacy and safety of ticagrelor versus clopidogrel in Asian patients $(n=1106)$ were similar to non-Asian patients $(n=17,515)$ [16]. However, clinical data with DAPT in Asian patients is limited, and Asian patients are hypothesized to have different risk profiles for thrombophilia and bleeding versus Caucasian patients [17]. Furthermore, clopidogrel requires metabolic activation via several cytochrome P450 (CYP) isoenzymes, particularly CYP2C19 [18]. CYP2C19 loss-offunction alleles result in clopidogrel resistance [19], and the prevalence of these alleles is high in Chinese patients (e.g., up to $60 \%$ [20-22]). Patients with CYP2C19 loss-of-function alleles treated with clopidogrel are at high risk of adverse cardiovascular outcomes $[19,21]$. Thus, alternative drugs are of high interest in Chinese ACS patients needing alternative DAPT, such as ticagrelor [20,22]. The mean bioavailability of ticagrelor and AR-C124910XX (the active metabolite of ticagrelor) is higher $(\sim 20-40 \%)$ in Asian subjects versus Caucasians, although this difference is unlikely to be clinically relevant [23-26]. Thus, further clinical data with ticagrelor at the approved dosing regimen in Chinese patients with ACS are required.

In agreement with the Chinese Food and Drug Administration (CFDA), the present DAYU study was conducted to further assess the safety profile of ticagrelor in a large population of Chinese patients with ACS. The primary objective was to describe the safety and tolerability of ticagrelor by assessing bleeding events and other serious adverse events (SAEs) during 1-year follow-up. The secondary objectives were to describe the incidence of CV events (including CV death, MI, stroke) with ticagrelor during 1-year follow-up in Chinese ACS patients, and to explore the incidence of fatal/life-threatening bleeding and major bleeding in various patient subgroups.

\section{Methods}

\section{Study Design}

The DAYU study (NCT01870921) was an interventional, open-label, multicenter, single-arm, phase IV trial conducted in China. Patients were enrolled on the first day of hospitalization following the index ACS event. The treatment approach for the index ACS event (i.e., invasive therapy or medical management) was at the discretion of the local investigator. Patients were also treated with ticagrelor (180 mg loading dose followed by $90 \mathrm{mg}$ b.i.d), plus low-dose aspirin (75$100 \mathrm{mg} /$ day), for 12 months. Given that the ACS patients were in a critical condition and in keeping with current guidelines for clinical management of ACS, initiation of the study drug (ticagrelor) took place as soon as possible after randomization and was not delayed to allow procedures (including laboratory testing and angiograms) to be conducted. Ticagrelor was supplied to patients, who returned all unused study medication/ empty packages at each visit for assessment of treatment compliance by pill count. 
The study was conducted in accordance with the Declaration of Helsinki and the International Conference on Harmonization/Good Clinical Practice (GCP) Guidelines, and followed applicable regulatory requirements including AstraZeneca's policy on bioethics. The local Institutional Review Boards or Independent Ethics Committees approved the final protocol and amendment. Of the 104 sites that participated in the DAYU study, 93 (89.4\%) were CFDA-GCP certified clinical trial institutions. Such institutions obtain certification from the state CFDA prior to conducting clinical trials. The CFDA monitors site personnel and corresponding facilities to ensure that the institution is appropriate for conducting clinical trials on drugs for human use. After completion of the study, the study operation team conducted the selfverification process at 84 sites and for 1575 patients, as per the CFDA guidance (i.e., prior to on-site inspection requirements introduced in 2016). Quality-control approval and certified site summaries were received from all the 104 participating investigator sites. There were no external inspections of the study sites or data.

\section{Patients}

Eligible patients were ethnic Chinese men and women (nonpregnant, or post-menopausal, or surgically sterile) aged $\geq 18$ years, with an index ACS event of NSTEMI, STEMI, or unstable angina. Qualifying events met the following criteria: hospitalization with cardiac ischemic symptoms for $\geq 10 \mathrm{~min}$ at rest and either (1) persistent ST segment elevation $\geq 1 \mathrm{~mm}(0.1 \mathrm{mV})$ in two or more contiguous leads and primary percutaneous coronary intervention (PCI) planned, or (2) new or presumed new left bundle branch block and primary PCI planned, or (3) ST segment changes on electrocardiogram (ECG) indicative of ischemia and/or positive biomarker evidence of myocardial necrosis.

Major exclusion criteria reflected contraindications and precautions detailed in the ticagrelor prescribing information and included contraindications to ticagrelor (hypersensitivity, active bleeding, moderate or severe liver disease, history of intracranial bleed, major surgery within 30 days); planned urgent $\mathrm{CABG}$ within 7 days from enrolment; use of non-selective, non-steroidal anti-inflammatory drugs that could not be stopped; or oral anticoagulation therapy within 30 days of enrolment or that could not be stopped. Concomitant therapy with strong CYP $3 \mathrm{~A}$ inhibitors, CYP3A substrates with narrow therapeutic indices, or strong CYP3A inducers was prohibited.

In addition to fulfilling eligibility criteria, all patients were required to accept study participation in the acute situation by providing written informed consent before any study-related procedures were initiated.

\section{Safety Assessments}

Patients returned to the clinic at 6 weeks, 3, 6, 9, and 12 months after start of study drug for assessments of adverse events (AEs) and concomitant medication. AEs were also collected in medical records, and these records were checked during the study clinic visits and during the monitoring visits as well as during site self-inspection. In addition, physical examinations, vital signs and safety laboratory measurements were included at 6 weeks and at 12 months (or at the end of treatment, if earlier). Laboratory assessments could be conducted at any visit at the discretion of the investigator. A follow-up visit was scheduled 2 weeks after the end of treatment.

AEs and SAEs, including bleeding, were recorded by the local investigators according to standard classifications used in clinical studies. These events were classified by the investigators as mild (i.e., awareness of sign or symptom, which is easily tolerated), moderate (i.e., discomfort sufficient to cause interference with normal activities), or severe (i.e., incapacitating, and inability to perform normal activities). All bleeding events were also categorized by the local investigators according to the definitions used in the PLATO study [14, 27] (Supplementary methods of Supplementary Material Online).

\section{Cardiovascular Events}

Clinical endpoints were evaluated by local investigators and included CV death (death from CV or cerebrovascular causes), MI (elevation of myocardial necrosis biomarkers with $\geq 1$ of the following: recurrent cardiac ischemic symptoms, development of new pathological Q waves in the ECG, and new or presumed new ECG changes indicative of ischemia), and stroke (a neurological deficit caused by an ischemic or hemorrhagic central nervous system event with residual symptoms for $\geq 24 \mathrm{~h}$ after onset or leading to death). Ischemic CV events were not reported as AEs.

\section{Sample Size and Data Analyses}

In PLATO, the risk of fatal/life-threatening bleeding in the Chinese subgroup was 5.7\% [AstraZeneca data on file] and similar to the risk of 5.8\% in the full cohort [14]. Thus, 2000 patients would be required for the rate of fatal/life-threatening bleeding to be estimated with a precision of $1 \%(95 \% \mathrm{CI})$ in the current study.

A patient was considered to be compliant with ticagrelor treatment if $>80$ and $<120 \%$ of the planned number of tablets were taken during their treatment period (excluding any days when the study drug was temporarily stopped). Safety data were summarized descriptively for the safety population. Frequency and percentage of patients in each category for PLATO-defined bleeding events, AEs, SAEs, and AEs of 
special interest (dyspnea and hyperuricemia) were tabulated for events reported during ticagrelor treatment, unless otherwise indicated. An exploratory analysis of fatal/lifethreatening bleeding events was conducted for the following subgroups: sex (males vs. females), age ( $<75$ years vs. $\geq 75$ years), use of glycoprotein (GP)IIb/IIIa inhibitors (yes vs. no), and treatment (medically managed vs. invasive therapy). For time-to-major CV event (composite of CV death/MI/ stroke), Kaplan-Meier (KM) plots were constructed, and the $\mathrm{KM}$ estimate and $95 \% \mathrm{CI}$ for this endpoint, and the individual components at 12 months (365 days), were calculated.

\section{Results}

\section{Patients and Treatment}

The DAYU study was conducted across 104 investigational centers in 21 provinces or municipalities of China (Participating sites in Supplementary Material Online) between 26 June 2013 (first patient enrolled) and 15 September 2015 (last patient completed the study).

Overall, 2004 patients provided written, informed consent, 2001 received ticagrelor and constituted the safety population (Fig. 1). Of these patients, 1939 were eligible for study inclusion. The remaining 62 patients (3\% of safety population) received ticagrelor despite not fulfilling eligibility criteria, most commonly due to the final diagnosis of the index event not meeting study STEMI or NSTEMI criteria. Consistent with current medical guidelines on antiplatelet therapies, the study protocol required patients to be enrolled as quickly as possible after presentation in order to maximize potential clinical benefits of treatment. Therefore, in these cases, it is likely that as medical assessment continued and more information became available, the investigator made relevant adjustments to the final diagnosis. A total of 1651 patients (82.5\% of safety population) completed study treatment (i.e., patients who continued ticagrelor for 12 months or until death), while 350 patients of the safety population prematurely terminated treatment, primarily due to patient decision (9.3\%), AEs (4.6\%), and non-compliance (1.4\%). Of the 29 patients who discontinued the study due to severe non-compliance to the protocol, six patients had nine AEs reported during the last month prior to discontinuation, no SAEs were reported. The nine reported AEs were anemia, diarrhea, non-cardiac chest pain, epistaxis, blurred vision, nausea, vomiting, abdominal pain, and urticaria. None of these AEs resulted in treatment discontinuation in these patients. Of the patients who prematurely terminated study treatment, 67 patients were given clopidogrel as an alternative antiplatelet agent (no other antiplatelet agents were used post-discontinuation).

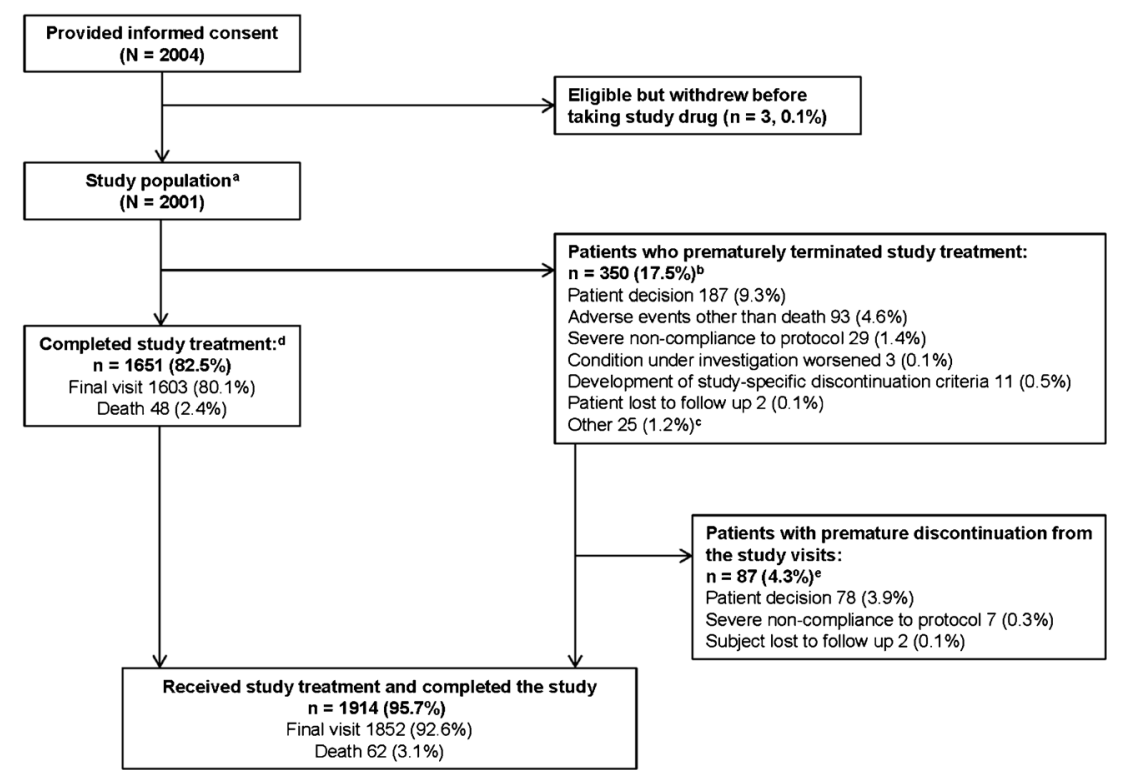

Fig. 1 Patient disposition. a Sixty-two patients failed screening as they did not meet inclusion criteria and received study medication. These patients are included in the safety analyses. b For patients who prematurely discontinued the study drug, the study visits continued, if these were acceptable to the patient. c Other reasons for early study termination included: enrollment error $(n=6)$, planned/conducted coronary artery bypass grafting $(n=5)$, angiogram results indicated no need for antiplatelet therapy $(n=5)$, patient withdrew consent $(n=3)$, investigator decision $(n=3)$, reduced bleeding risk $(n=1)$, imprisonment $(n=1)$, and car accident $(n=1)$. $\mathbf{d}$ Completion of study treatment was defined as treatment continuation up to the final visit or death, whichever occurred first. e Completion of study visits was defined as completion of the visits up to the final visit or death, whichever occurred first 
Table 1 shows the baseline characteristics of the safety population. Overall, 1999 (99.9\%) patients had at least one concomitant medication, most commonly aspirin (99.2\%), HMG-CoA reductase inhibitors $(95.3 \%)$, or heparin $(81.4 \%)$.

Mean (standard deviation [SD]) treatment compliance was $98.5 \%$ (9\%). Mean (SD) exposure to ticagrelor was 309 (126) days. Overall, 82.6 and $81.2 \%$ of patients had $>6$ or $>9$ months of exposure, respectively. Some patients received ticagrelor treatment for $>12$ months; $13.4 \%$ received ticagrelor for $>12-\leq 13$ months, $0.4 \%$ for $>13-\leq 14$ months, and $0.1 \%$ for $>14$ months.

Table 1 Patient demographics and disease characteristics (safety population)

\begin{tabular}{ll}
\hline Characteristic & $\begin{array}{l}\text { Number (\%) of patients, } \\
\text { unless otherwise stated } \\
(n=2001)\end{array}$ \\
\hline Age, mean (range), years & $59.3(25-96)$ \\
Age group & \\
$<75$ years & $1854(92.7)$ \\
$\geq 75$ years & $147(7.3)$ \\
Sex & \\
Male & $1579(79)$ \\
Female & $422(21)$ \\
BMI, mean (SD), kg/m ${ }^{2}$ & $24.8(3.3)$ \\
Race & \\
Chinese & $2001(100)$ \\
Medical history & \\
Hypertension & $1102(55.1)$ \\
Coronary artery disease & $686(34.3)$ \\
Angina pectoris & $656(32.8)$ \\
Diabetes & $520(26.0)$ \\
Dyslipidemia & $380(19.0)$ \\
Peptic ulcer disease & $96(4.8)$ \\
Gout & $29(1.4)$ \\
Chronic obstructive & $19(0.9)$ \\
pulmonary disease & \\
Final diagnosis of index event & \\
Unstable angina pectoris & \\
ST elevation MI & $488(24.4)$ \\
Non-ST elevation MI & $1064(53.2)$ \\
Invasive therapy & $424(21.2)$ \\
Medically managed therapy & $25(1.2)$ \\
\hline
\end{tabular}

$B M I$ body mass index, $M I$ myocardial infarction, $S D$ standard deviation

\section{Bleeding AEs}

Overall, of the safety population, 426 (21.3\%) patients experienced at least one bleeding $\mathrm{AE}$ (see the next section for bleeding events classified according to PLATO definition) (Table 2), and $422(21.1 \%)$ had bleeding AEs during treatment. On-treatment bleeding AEs were mild (17.0\%), moderate $(2.4 \%)$, or severe $(1.7 \%)$. Fifty-four $(2.7 \%)$ patients experienced at least one bleeding SAE. Seven, 15 , and 32 patients experienced mild, moderate, or severe bleeding SAEs, respectively, during treatment. On-treatment, bleeding AEs necessitated ticagrelor discontinuation in $55(2.7 \%)$ patients. Treatment-related bleeding AEs (per investigator judgment) were reported in 294 (14.7\%) patients; 41 (2.0\%) patients had treatment-related SAEs.

\section{Bleeding Events Classified According to PLATO Definition}

PLATO-defined bleeding events during and post-treatment combined are shown in Table 3. PLATO-defined major, minor, and minimal bleedings (composite) occurred in 426 $(21.3 \%)$ patients, the majority of whom $(n=333)$ had minimal bleeding only.

Among PLATO-defined major bleeding events, fatal/lifethreatening bleeding was observed in $17(0.8 \%)$ patients, with a KM estimated event risk (95\% CI) of 1.0\% (0.6-1.6). Fourteen patients experienced fatal/life-threatening spontaneous bleeding and three patients had traumatic bleeding; the two most common bleeding sites were gastrointestinal tract $(n=10)$ and intracranial system $(n=4)$. Overall, four of these events were fatal and none were procedurally related. Other major bleeding events were reported in $11(0.55 \%)$ patients, with one patient experiencing both fatal/life-threatening and other major bleeding events. The KM estimated event risk $(95 \% \mathrm{CI})$ for the 27 patients who experienced fatal/life-threatening bleeding and/or other major bleeding was $1.6 \%(1.1-2.3)$.

Minor bleeding occurred in 66 (3.3\%) patients, with a KM estimated event risk (95\% CI) of 3.7\% (2.9-4.7). For the 93 patients with major or minor bleeding, the KM estimated event risk (95\% CI) was 5.2\% (4.3-6.4).

\section{Exploratory Analyses of PLATO-Defined Bleeding Events}

Exploratory analyses of PLATO-defined bleeding events by patient subgroup showed that all 17 fatal/life-threatening events occurred in men $<75$ years old, i.e., the largest age and gender subgroup. There was no notable difference in the number of patients experiencing fatal/life-threatening events between patients on invasive therapy versus those being 
Table 2 Bleeding adverse events (overall incidence $\geq 0.2 \%$ ) by causality and preferred term (safety population)

\begin{tabular}{|c|c|c|c|}
\hline \multirow[t]{2}{*}{ Preferred term } & \multicolumn{3}{|c|}{$\begin{array}{l}\text { Ticagrelor } 90 \mathrm{mg} \text { b.i.d } \\
n=2001\end{array}$} \\
\hline & $\begin{array}{l}\text { Related to } \\
\text { treatment } n(\%)\end{array}$ & $\begin{array}{l}\text { Not related to } \\
\text { treatment } n(\%)\end{array}$ & Overall $n(\%)$ \\
\hline Patients with at least one bleeding $\mathrm{AE}$ & $296(14.8)^{\mathrm{a}}$ & $130(6.5)$ & $426(21.3)$ \\
\hline Ecchymosis & $83(4.1)$ & $27(1.3)$ & $110(5.5)$ \\
\hline Gingival bleeding & $68(3.4)$ & $23(1.1)$ & $91(4.5)$ \\
\hline Epistaxis & $46(2.3)$ & $32(1.6)$ & $78(3.9)$ \\
\hline Subcutaneous hemorrhage & $24(1.2)$ & $16(0.8)$ & $40(2.0)$ \\
\hline Upper gastrointestinal hemorrhage & $18(0.9)$ & $4(0.2)$ & $22(1.1)$ \\
\hline Gastrointestinal hemorrhage & $12(0.6)$ & $7(0.3)$ & $19(0.9)$ \\
\hline Hemoptysis & $10(0.5)$ & $5(0.2)$ & $15(0.7)$ \\
\hline Petechiae & $10(0.5)$ & $2(0.1)$ & $12(0.6)$ \\
\hline Hematuria & $6(0.3)$ & $4(0.2)$ & $10(0.5)$ \\
\hline Hematochezia & $5(0.2)$ & $3(0.1)$ & $8(0.4)$ \\
\hline Contusion & $6(0.3)$ & $1(0.0)$ & $7(0.3)$ \\
\hline Occult blood positive & $3(0.1)$ & $3(0.1)$ & $6(0.3)$ \\
\hline Conjunctival hemorrhage & $4(0.2)$ & $1(0.0)$ & $5(0.2)$ \\
\hline Cerebral hemorrhage & $3(0.1)$ & $1(0.0)$ & $4(0.2)$ \\
\hline Hemorrhoidal hemorrhage & $3(0.1)$ & $1(0.0)$ & $4(0.2)$ \\
\hline Retinal hemorrhage & $2(0.1)$ & $2(0.1)$ & $4(0.2)$ \\
\hline Urethral hemorrhage & $3(0.1)$ & $1(0.0)$ & $4(0.2)$ \\
\hline
\end{tabular}

${ }^{\text {a }}$ Causality assessment as judged by the investigator. One patient had an AE of epistaxis before start of study treatment which was reported as related to the study drug as this patient was prescribed ticagrelor prior to enrolment. This summary table includes events regardless if the onset was during or after treatment with ticagrelor $A E$ adverse event, b.i.d twice daily

Table 3 PLATO-defined bleeding events by severity (safety population)

\begin{tabular}{lll}
\hline Bleed severity & $\begin{array}{l}\text { Ticagrelor } 90 \mathrm{mg} \text { b.i.d } \\
(n=2001)\end{array}$ \\
\cline { 2 - 3 } & $\begin{array}{l}\text { Patients with } \\
\text { bleeding } \\
n(\%)\end{array}$ & $\begin{array}{l}\text { Number of } \\
\text { bleeding events }\end{array}$ \\
& $27(1.3)$ & 28 \\
\hline Total major bleeding & $17(0.8)$ & 17 \\
Life-threatening/fatal & $4(0.2)$ & 4 \\
$\quad$ Fatal & $13(0.6)$ & 13 \\
$\quad$ Life-threatening & $11(0.5)$ & 11 \\
Major, other & $93(4.6)$ & 106 \\
Composite of major \\
$\begin{array}{l}\text { and minor bleeding } \\
\text { Minor bleeding }\end{array}$
\end{tabular}

This summary table includes all recorded events regardless of timing relative to study treatment

b.i.d twice daily medically managed. The proportion of patients experiencing major bleeding events (including fatal/life-threatening events) was similar between males and females (1.4 vs. $1.2 \%$, respectively), and between patients receiving GPIIb/IIIa inhibitors versus those who were not (1.4 vs. $1.2 \%$, respectively). Major bleeding events were reported in three patients aged $\geq 75$ years $(2.0 \%)$ versus in 24 patients aged $<75$ years $(1.3 \%)$, and in five patients who received medically managed therapy versus 22 receiving invasive therapy (2.0 vs. $1.3 \%$, respectively).

\section{Non-bleeding AEs and SAEs}

Of the safety population, 784 (39.2\%) patients experienced at least one non-bleeding AE during treatment (Table S1 of Supplementary Material Online). The most common, nonbleeding events during treatment were hyperuricemia and dyspnea. Most patients reported non-bleeding AEs during treatment that were mild in intensity ( $28.4 \%$ of safety population), and only $4.0 \%$ of patients reported a severe event. During treatment, $39(1.9 \%)$ patients discontinued study treatment due to a non-bleeding AE and 197 (9.8\%) patients reported non-bleeding AEs that were considered to be treatment related by the local investigator. 
Overall, $116(5.8 \%)$ patients reported a non-bleeding SAE during treatment (Table S2 of Supplementary Material Online). The two most common, non-bleeding SAEs during treatment were chest discomfort $(n=6,0.3 \%)$ and cardiac failure $(n=5,0.2 \%)$. In total, $13(0.6 \%)$ patients died due to a non-bleeding SAE, four $(0.2 \%)$ discontinued study treatment due to a non-bleeding SAE, and $11(0.5 \%)$ experienced events that were considered to be treatment related.

\section{AEs of Special Interest: Dyspnea and Hyperuricemia/Gout}

Dyspnea AEs were reported in 68/2001 (3.4\%) patients during ticagrelor treatment (Table S3 of Supplementary Material Online). Of these patients, 53 experienced mild dyspnea AEs. Dyspnea SAEs occurred in two patients during treatment and no patients died due to dyspnea. AEs related to hyperuricemia were reported in $130(6.5 \%)$ patients during treatment, and gout was reported in eight $(0.4 \%)$ patients (Table S3 of Supplementary Material Online). Most patients reported mild events during treatment $(163,8.1 \%)$. There were no reports of urate nephropathy. Three patients experienced hyperuricemia or gout SAEs during treatment and no event was fatal. Two patients discontinued the trial due to hyperuricemia/gout, and 86 patients experienced hyperuricemia or gout considered treatment-related by the investigator.

\section{AEs Related to Hepatic Function}

During treatment, abnormal hepatic function AEs were recorded in 51/2001 (2.6\%) patients (Table S3 of Supplementary Material Online). Of these patients, 44 reported a mild event, six reported a moderate event, and one reported a severe event. Most of these AEs were based on changes in aspartate aminotransferase (AST), alanine aminotransferase (ALT), and bilirubin, with liver function tests being higher than the upper limit of normal range at baseline; AST and ALT then decreased during the study. In the fullstudy cohort, mean (SD) AST levels decreased over time from $1.36(2.01) \mu \mathrm{kat} / \mathrm{L}$ at visit 1 to 0.51 (3.87) $\mu \mathrm{kat} / \mathrm{L}$ at the end of treatment, with a similar pattern for ALT. The severe AE was an SAE and was considered to be treatment-related by the investigator, thus, this patient discontinued the study. Overall, eight patients experienced treatment-related, abnormal hepatic function as judged by the investigator.

\section{Cardiovascular Events}

In the safety population, 83 patients had a major CV event (i.e., composite of CV death, MI, or stroke) within 12 months after first exposure to ticagrelor, with a $\mathrm{KM}$ estimated event risk $(95 \% \mathrm{CI})$ of $4.3 \%$ (3.5-5.3). Approximately $50 \%$ of the major $\mathrm{CV}$ events occurred during the first 6 weeks from the start of ticagrelor at the index event (Fig. 2). Of the safety population (KM estimated 12-month event risk [95\% CI]), CV death occurred in 50 patients $(2.6 \%$ [2.0-3.4]), and stroke occurred in 23 patients $(1.2 \%$ [0.8-1.8]). A total of $21 \mathrm{MI}$ events (NSTEMI $n=11$, STEMI $n=8$ ) occurred in 19 patients (KM estimated 12-month event risk [95\% CI]: 0.9\% [0.6-1.4]).

Of the 350 patients who discontinued ticagrelor before 12 months, a similar proportion of patients experienced major CV events $(5.4 \%, 19 / 350)$ compared with the overall population $(4.1 \%, 83 / 2001)$. For the 19 patients who discontinued ticagrelor and had a major CV event, 11 had the event while still on treatment and eight experienced the event after treatment discontinuation and within 12 months of study start.
Fig. 2 Kaplan-Meier plot of the first risk (cumulative incidence) of major cardiovascular event* (safety population). * a major cardiovascular (CV) event is defined as a composite of $\mathrm{CV}$ death, myocardial infarction, and stroke

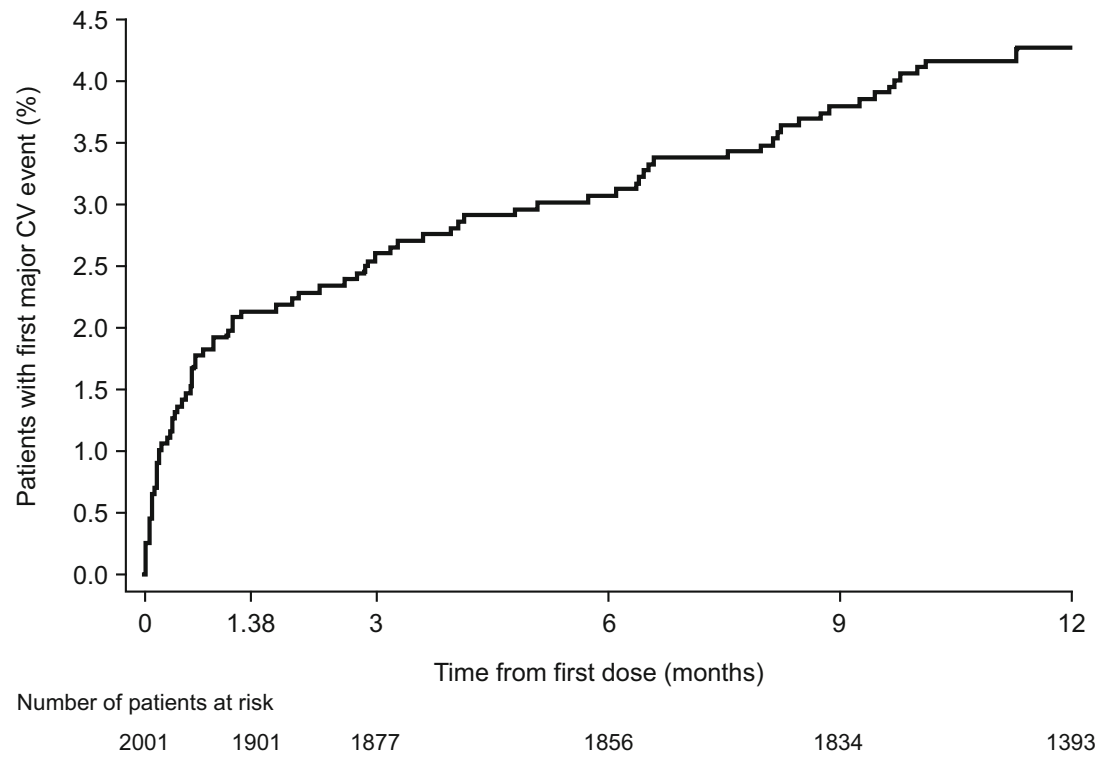


For patients treated with invasive therapy $(n=1756)$, 66 patients had a major $\mathrm{CV}$ event within 12 months after first exposure to ticagrelor, KM estimated 12-month event risk $(95 \% \mathrm{CI})$ was $3.8 \%(3.0-4.9)$. Of the 245 patients who were medically managed, 17 patients had a major $\mathrm{CV}$ event within 12 months after first exposure to ticagrelor, $\mathrm{KM}$ estimated 12 -month event risk $(95 \% \mathrm{CI})$ was $7.5 \%(4.7-11.8)$.

\section{Discussion}

The DAYU study described the safety and rate of CV events during ticagrelor treatment in a large cohort of Chinese ACS patients, a population with limited data when ticagrelor was approved in China in 2012. The safety of ticagrelor in Chinese patients had been evaluated in major trials which included small numbers of Asian patients [14-16]. However, DAYU is the first study to describe clinical experience with ticagrelor in a larger group of Chinese ACS patients. The DAYU study demonstrated that ticagrelor $90 \mathrm{mg}$ twice daily with low-dose aspirin for up to 1 year is associated with a low incidence of major bleeding events and SAEs, and a low incidence of major $\mathrm{CV}$ events in Chinese patients with ACS.

These findings are clinically important for several reasons. Firstly, as a phase IV study, the study population was aligned with the ticagrelor label in China and reflective of current clinical practice in this region (e.g., eligibility for ticagrelor treatment requires that patients with ACS must not have planned CABG within 7 days, must not have a history of intracranial bleeding, and must have no active bleeding). Secondly, the planned (12 months) and reported (309 days, $\sim 10.2$ months) mean duration of ticagrelor exposure in the DAYU study were aligned with label recommendations for ticagrelor in ACS patients [13] and international guidelines [5-9], and a high proportion of patients completed treatment. Additionally, demographic and baseline characteristics, medical history, and index event interventions in the DAYU study were similar to those observed in a real-world study in China (e.g., Chinese subgroup in the EPICOR Asia study [3]). In fact, the study population in the DAYU study was similar in characteristics, considering age, gender, STEMI frequency, and management strategy, to that included in the International EPICOR registry study [28].

Bleeding is the most common, clinically significant, safety concern during effective antiplatelet treatment, due to the mechanism of action [5-9, 29]. In the DAYU study, the incidence of PLATO-defined fatal/life-threatening bleeding and major bleeding in Chinese patients with ACS receiving ticagrelor was low (0.8 and $1.3 \%$, respectively). Furthermore, the majority of bleeding events was minimal and did not require intervention. This pattern of bleeding events was consistent with previous ticagrelor data $[14,16,30]$. The low incidence of fatal bleedings $(0.2 \%)$ in DAYU was consistent with those reported in the PLATO overall population $(0.3 \%, 20 / 9235$ [14]) and the PLATO Asian subpopulation (0.4\%, 5/548 [16]), while reports of fatal/life-threatening bleedings were less frequent (0.8\% [DAYU], 5.8\% [PLATO overall] [14]). Furthermore, the incidence of major CV events was also lower in DAYU than observed with ticagrelor in PLATO [14], PLATO Asian substudy [16], and PHILO [30].

For non-bleeding events, the incidence of SAEs was generally low in the DAYU study. As expected in ACS patients, the most common non-bleeding SAEs were cardiac disorders. The two most commonly reported nonbleeding AEs were dyspnea and hyperuricemia, consistent with previous ticagrelor studies and the current ticagrelor prescribing information [13-15, 30, 31]. The incidence of dyspnea and hyperuricemia was low, and neither event was fatal. The dyspnea rate in DAYU (3.4\%) was similar to that in a ticagrelor study in Japanese, Korean, and Taiwanese patients with ACS $(5.7 \% ; 22 / 401)$ [30], but lower than in the PLATO overall cohort $(13.8 \%$; 1270/9235) [14] or PLATO Asian subcohort [11.6\%; 16]. The third most common AE in the DAYU study was abnormal hepatic function, which is not an AE associated with ticagrelor therapy [13]. In most cases, high levels of AST and ALT were recorded at baseline, which is expected after an ACS event. Elevated hepatic enzymes declined during continued ticagrelor treatment, which indicate a relationship to the background disease rather than ticagrelor. However, this observation highlights one key limitation in that the DAYU study was uncontrolled. Thus, the influence of confounding factors is more difficult to assess in the absence of a placebo arm. Of note, no patient in the DAYU study met the criteria for Hy's Law at any time.

When contextualizing the above findings, important key differences between the DAYU and PLATO patient populations should be noted. Compared with PLATO patients, the DAYU population was generally younger, had fewer females, and exhibited fewer CV risk factors. In addition, PLATO and DAYU were conducted during different time periods, with PLATO starting in 2006 and DAYU commencing 7-8 years later, which may help explain why $\mathrm{CV}$ event rates are lower in the latter study due to management progression of these patients. Furthermore, as the DAYU study started after ticagrelor approval in China, recruited patients met the criteria of ACS patients described in the Chinese ticagrelor label, e.g., patients with planned CABG were excluded from DAYU, but were included in PLATO [14], and the described dyspnea side effect in the Chinese ticagrelor label may have impacted the recruitment of patients at risk for 
such symptoms. Exploratory analyses of the incidence of fatal/life-threatening bleeding and major bleeding in the DAYU study indicated that such events were generally consistent across the various patient subgroups assessed. However, it should be noted that the low incidence of bleeding in these categories and the imbalance in size of some subgroups make it difficult to draw firm, risk-related conclusions.

Overall, no new or unexpected safety findings were observed in the DAYU study, and the observed profile of ticagrelor reflected current Chinese prescribing information [13]. The safety profile with ticagrelor in Chinese patients with ACS was comparable to that reported in other large ticagrelor trials in a wide variety of patients $[14,15,30,32,33]$.

There are several study limitations. DAYU was a singlearm, non-randomized study with no control arm, the design of which does not allow direct comparisons with other treatments. Hence, the results of the DAYU study were presented descriptively. However, this design was appropriate for the purpose of describing the safety of ticagrelor in a large population of contemporary Chinese patients experiencing an ACS event. An endpoint adjudication committee was not appointed to assess clinical outcomes in DAYU, which could be interpreted as a potential source of reporting bias. The potential risk of underreporting of AEs or CV events was mitigated by monitoring of participating sites. Although the DAYU study investigated the safety and incidences of CV events when used in a broad range of hospitals and in line with the Chinese prescribing information, it is still an interventional study where study drug was supplied to patients, which may have impacted treatment compliance. Patients with known or suspected contraindications to ticagrelor were excluded from the study, which may have resulted in a population with a lower risk of experiencing AEs commonly associated with this treatment (e.g., dyspnea) than in earlier studies. However, since the eligibility criteria were developed in line with the approved ticagrelor label, the DAYU study population should better reflect the patient population receiving ticagrelor in real life. Although a potential bias of this study is that patients were required to be enrolled as quickly as possible after presentation with ACS; therefore, it would not have been possible to enroll patients dying very early on presentation.

In conclusion, overall, the DAYU study demonstrated that exposure to ticagrelor plus aspirin for up to 1 year in a large population of Chinese patients with ACS resulted in a low rate of PLATO-defined major bleeding. The overall safety profile was in keeping with the current prescribing information and no new safety findings were found. Furthermore, the incidence of major $\mathrm{CV}$ events (CV death, MI, and stroke) was low during the study.
Acknowledgments The authors wish to thank the patients who participated in the DAYU study, the site management organization by PRIMIUM Site Management Co., Ltd., the data management and statistics services provided by Quintiles Medical Development (Shanghai) Co., Ltd., and the clinical research organization CGP CMIC ClinPlus Co., Ltd., for their support in the conduct of this study. The authors thank the following individuals for their contribution to the study: Wei Miao, Guoying Geng, Wenjie Han, and Lei Wu. Medical writing assistance was provided by Lisa Michel, $\mathrm{PhD}$ and Jackie Phillipson, $\mathrm{PhD}$ (both of Zoetic Science, an Ashfield company, part of UDG Healthcare plc, Macclesfield, UK); this assistance was funded by AstraZeneca.

Funding This study was funded by AstraZeneca.

\section{Compliance with Ethical Standards}

Conflict of Interest $\mathrm{RG}$ received a research grant from AstraZeneca. HL, YWu, GS, Yan W, ZW, HZ, AZ, YZ, Yong W, ZY, and YH declare no conflicts of interest. LL and ML-Z are current employees of AstraZeneca. LS was an employee of AstraZeneca when the study was conducted.

Ethical Approval The study was conducted in accordance with the Declaration of Helsinki and the International Conference on Harmonization/Good Clinical Practice Guidelines, and followed applicable regulatory requirements including AstraZeneca's policy on bioethics. The local Institutional Review Boards or Independent Ethics Committees approved the final protocol and amendment. Written informed consent was obtained from all patients.

Abbreviations ACS, acute coronary syndrome; AE, adverse event; ALT, alanine aminotransferase; AST, aspartate aminotransferase; b.i.d, twice daily; CABG, coronary artery bypass grafting; CFDA, Chinese Food and Drug Administration; CI, confidence interval; CV, cardiovascular; CVD, cardiovascular disease; DAPT, dual antiplatelet therapy; ECG, electrocardiogram; GPIIb/IIIa, glycoprotein IIb/glycoprotein IIIa; KM, Kaplan-Meier; MI, myocardial infarction; NSTEMI, non-ST elevation myocardial infarction; PCI, percutaneous coronary intervention; SAE, serious adverse event; SD, standard deviation; STEMI, ST elevation myocardial infarction

Open Access This article is distributed under the terms of the Creative Commons Attribution 4.0 International License (http:// creativecommons.org/licenses/by/4.0/), which permits unrestricted use, distribution, and reproduction in any medium, provided you give appropriate credit to the original author(s) and the source, provide a link to the Creative Commons license, and indicate if changes were made.

\section{References}

1. Li H, Ge J. Cardiovascular disease in China: current status and future perspectives. Int J Cardiol Heart Vasc. 2015;6:25-31.

2. Chan MY, Du X, Eccleston D, et al. Acute coronary syndrome in the Asia-Pacific region. Int J Cardiol. 2016;202:861-9.

3. Huo Y, Han YL, Ge J, et al. Two-year outcomes post discharge in Chinese patients with acute coronary syndrome: findings from the EPICOR Asia study. J Am Coll Cardiol. 2016;67(13):505.

4. Wang W, Zhao D, Liu J, Qi Y, Sun J, Liu J. The prevalence of risk factors and status of clinical practice patterns among hospitalized patients with acute coronary syndromes. Zhonghua Nei Ke Za Zhi. 2014;53(8):611-6. 
5. O'Gara PT, Kushner FG, Ascheim DD, et al. 2013 ACCF/AHA guideline for the management of ST-elevation myocardial infarction: a report of the American College of Cardiology Foundation/ American Heart Association Task Force on Practice Guidelines. Circulation. 2013;127(4):e362-425.

6. Steg PG, James SK, Atar D, et al. ESC guidelines for the management of acute myocardial infarction in patients presenting with STsegment elevation. Eur Heart J. 2012;33(20):2569-619.

7. Amsterdam EA, Wenger NK, Brindis RG, et al. 2014 AHA/ACC guideline for the management of patients with non-ST-elevation acute coronary syndromes: executive summary: a report of the American College of Cardiology/American Heart Association Task Force on Practice Guidelines. Circulation. 2014;130(25): 2354-94.

8. Roffi M, Patrono C, Collet JP, et al. 2015 ESC guidelines for the management of acute coronary syndromes in patients presenting without persistent ST-segment elevation: Task Force for the Management of Acute Coronary Syndromes in Patients Presenting without Persistent ST-Segment Elevation of the European Society of Cardiology (ESC). Eur Heart J. 2016;37(3):267-315.

9. China Society of Cardiology of Chinese Medical Association. Guideline on the diagnosis and therapy of ST-segment elevation myocardial infarction. Zhonghua Xin Xue Guan Bing Za Zhi. 2015;43(5):380-93.

10. Husted S, van Giezen JJ. Ticagrelor: the first reversibly binding oral P2Y12 receptor antagonist. Cardiovasc Ther. 2009;27(4):259-74.

11. van Giezen JJ, Nilsson L, Berntsson P, et al. Ticagrelor binds to human P2Y(12) independently from ADP but antagonizes ADPinduced receptor signaling and platelet aggregation. $\mathrm{J}$ Thromb Haemost. 2009;7(9): 1556-65.

12. Armstrong D, Summers C, Ewart L, Nylander S, Sidaway JE, van Giezen JJ. Characterization of the adenosine pharmacology of ticagrelor reveals therapeutically relevant inhibition of equilibrative nucleoside transporter 1. J Cardiovasc Pharmacol Ther. 2014;19(2): 209-19.

13. AstraZeneca LP. Brilinta (ticagrelor) tablets. Approved US prescribing information. Revised September 2016. Available at: http://www.azpicentral.com/brilinta/brilinta.pdf. Accessed 23 Oct 2017.

14. Wallentin L, Becker RC, Budaj A, et al. Ticagrelor versus clopidogrel in patients with acute coronary syndromes. N Engl $\mathrm{J}$ Med. 2009;361(11):1045-57.

15. Bonaca MP, Bhatt DL, Cohen M, et al. Long-term use of ticagrelor in patients with prior myocardial infarction. $\mathrm{N}$ Engl $\mathrm{J}$ Med. 2015;372(19):1791-800.

16. Kang HJ, Clare RM, Gao R, et al. Ticagrelor versus clopidogrel in Asian patients with acute coronary syndrome: a retrospective analysis from the Platelet Inhibition and Patient Outcomes (PLATO) Trial. Am Heart J. 2015;169(6):899-905.

17. Levine GN, Jeong YH, Goto S, et al. Expert consensus document: World Heart Federation expert consensus statement on antiplatelet therapy in East Asian patients with ACS or undergoing PCI. Nat Rev Cardiol. 2014;11(10):597-606.
18. Sangkuhl K, Klein TE, Altman RB. Clopidogrel pathway. Pharmacogenet Genomics. 2010;20(7):463-5.

19. Ma TKW, Lam Y-Y, Tan VP, Yan BP. Variability in response to clopidogrel: how important are pharmacogenetics and drug interactions? Br J Clin Pharmacol. 2011;72(4):697-706.

20. Guo YM, Zhao ZC, Zhang L, Li HZ, Sun HL. CYP2C19 polymorphisms in acute coronary syndrome patients undergoing clopidogrel therapy in Zhengzhou population. Genet Mol Res. 2016;15(2).

21. Xie X, Ma YT, Yang YN, et al. CYP2C19 phenotype, stent thrombosis, myocardial infarction, and mortality in patients with coronary stent placement in a Chinese population. PLoS One. 2013;8(3): e59344.

22. Tam CC, Kwok J, Wong A, et al. Genotyping-guided approach versus the conventional approach in selection of oral P2Y12 receptor blockers in Chinese patients suffering from acute coronary syndrome. J Int Med Res. 2017;45(1):134-46.

23. Li H, Butler K, Yang L, Yang Z, Teng R. Pharmacokinetics and tolerability of single- and multiple-doses of ticagrelor in healthy Chinese volunteers. Clin Drug Investig. 2012;32(2):87-97.

24. Teng R, Butler K. Pharmacokinetics, pharmacodynamics, and tolerability of single and multiple doses of ticagrelor in Japanese and Caucasian volunteers. Int J Clin Pharmacol Ther. 2014;52(6):47891.

25. Hiasa Y, Teng R, Emanuelsson H. Pharmacodynamics, pharmacokinetics and safety of ticagrelor in Asian patients with stable coronary artery disease. Cardiovasc Interv Ther. 2014;29(4):324-33.

26. Li H, Guo J, Carlson GF, Teng R. Pharmacodynamics, pharmacokinetics, and safety of ticagrelor in Chinese patients with stable coronary heart disease. Br J Clin Pharmacol. 2016;82(2):352-61.

27. James S, Akerblom A, Cannon CP, et al. Comparison of ticagrelor, the first reversible oral $\mathrm{P} 2 \mathrm{Y}(12)$ receptor antagonist, with clopidogrel in patients with acute coronary syndromes: rationale, design, and baseline characteristics of the PLATelet inhibition and patient Outcomes (PLATO) trial. Am Heart J. 2009;157(4):599-605.

28. Bueno H, Pocock S, Danchin N, et al. International patterns of dual antiplatelet therapy duration after acute coronary syndromes. Heart. 2017;103(2):132-8.

29. Bhatt DL, Hulot JS, Moliterno DJ, Harrington RA. Antiplatelet and anticoagulation therapy for acute coronary syndromes. Circ Res. 2014;114(12):1929-43.

30. Goto S, Huang CH, Park SJ, Emanuelsson H, Kimura T. Ticagrelor vs. clopidogrel in Japanese, Korean and Taiwanese patients with acute coronary syndrome - randomized, double-blind, phase III PHILO study. Circ J. 2015;79(11):2452-60.

31. Butler K, Teng R. Evaluation and characterization of the effects of ticagrelor on serum and urinary uric acid in healthy volunteers. Clin Pharmacol Ther. 2012;91(2):264-71.

32. Johnston S, Amarenco P, Albers G, et al. Ticagrelor versus aspirin in acute stroke or transient ischemic attack. N Engl J Med. 2016;375(1):35-43.

33. Hiatt WR, Fowkes GR, Heizer G, et al. Ticagrelor versus clopidogrel in symptomatic peripheral artery disease. $\mathrm{N}$ Engl $\mathrm{J}$ Med. 2017;376(1):32-40. 